\title{
EL PRINCIPIO TRIBUTARIO DE IGUALDAD, GENERALIDAD, PROPORCIONALIDAD Y LA SOCIEDAD EN EL PERÚ
}

\author{
THE PRINCIPLE OF EQUAL TAX, GENERALITY, \\ PROPORTIONALITY AND SOCIETY IN PERU \\ Raúl Mendoza Pérez * \\ Docente Auxiliar de la Facultad de Ciencias Contables
}

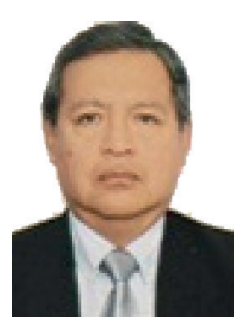

Universidad Nacional Mayor de San Marcos-UNMSM / Lima-Perú

[Recepción: Setiembre de 2014/ Conformidad: Octubre 2014]

\section{RESUMEN}

Comparar si los principios tributarios de igualdad, generalidad, proporcionalidad son aplicables y observados en los datos que proporcionan las autoridades del Estado peruano. En el presente trabajo, se utilizaron los datos encontrados en la Ley del Presupuesto Público para el año fiscal 2014, Superintendencia Nacional de Aduanas y de Administración Tributaria (SUNAT) aplicando el método deductivo. Asimismo, se encontró diferencias entre los principios tributarios y los datos proporcionados por el Estado y se sugiere que se invierta en las ciudades más pobres del Perú, considerando los ingresos ordinarios (tributos) importantes ya que representa el $70 \%$ del gasto público.

\section{Palabras clave:}

Igualdad; generalidad; proporcionalidad; progresividad; sociedad.

\begin{abstract}
Comparing if tax principles of equality, generality, and apply proportionality observed in the data provided by the authorities of the Peruvian State. In the present work, that were used from the data found in the Public Budget Act for fiscal year 2014, National Customs and Tax Administration (SUNAT) applying the deductive method. Also, differences between tax principles and data provided by the State and is suggested to invest in the poorest cities in Peru, considering the significant revenue (taxes) and representing $70 \%$ of public expenditure was found.
\end{abstract}

\section{Keywords:}

Equality; generality proportionality; progressivity; society.

* Contador Público Colegiado - UNMSM. E-mail: rmendozap@unmsm.edu.pe 


\section{INTRODUCCIÓN}

Si bien, en los últimos años la economía del Perú ha crecido y el sector público, ha destinado mayores recursos para solucionar los problemas de la agenda social, quedando pendientes asuntos por resolver que son atendidos por el Ministerio de Desarrollo e Inclusión Social (MIDIS). El mismo que fue creado por la Ley No 29792 el 20 de octubre de 2011. La juramentación de la primera Ministra del MIDIS se llevó a cabo del mismo año en la ciudad del Cusco el 21 de Octubre, siendo sus principales tareas:

- Igualdad de oportunidades. Lograr que los hogares en pobreza extrema o vulnerables accedan a los servicios públicos universales, ejerciendo así sus derechos y su ciudadanía plenamente a través de los programas de alivio temporales y focalizados.

- Fomentar capacidades para aprovechar oportunidades. Contribuir a que los usuarios de programas sociales logren estrategias sostenidas de generación de ingresos, seguridad alimentaria, reducción de vulnerabilidad y empleo que les permita - en el mediano plazo - superar su condición de pobreza y vulnerabilidad a través de la articulación de programas de alivio con programas promotores.

El objetivo es comparar los principios tributarios y su aplicación en la recaudación.

Se pretende apoyar el gran trabajo que realiza el Estado en busca de un desarrollo económico sostenido con igualdad de oportunidades.

Se ha desarrollado 8 conceptos:

1. El surgimiento del principio de igualdad.

2. Generalidad: Igualdad ante la ley.

3. La igualdad como base del tributo.

4. Distribución de las cargas públicas. Proporcionalidad y Progresividad.

5. Sociedad de personas.

6. Sociedad.

7. Ley del equilibrio financiero del presupuesto del sector público para el año fiscal 2014.

8. SUNAT (Superintendencia de Aduanas y de Administración tributaria). Estadística.

\section{EL SURGIMIENTO DEL PRINCIPIO DE IGUALDAD}

Referirse al principio de igualdad es hablar de uno de los principios básicos surgido de la Revolución Francesa, que impulsó la sustitución de un sistema basado en una sociedad clasista, por un ordenamiento jurídico sustentado en la igualdad de todos los hombres ante la ley.

Ilustración No 1

\section{LA REVOLUCIÓN FRANCESA IMPULSÓ}

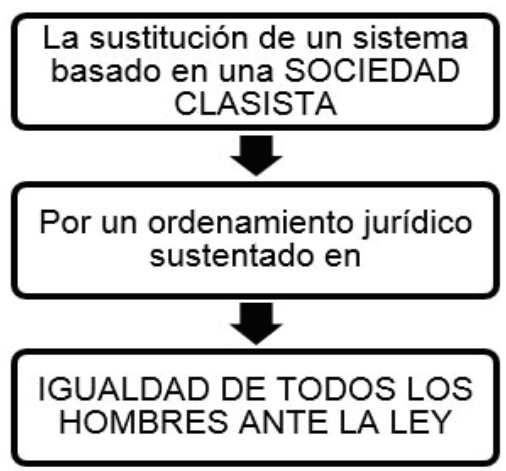

Fuente: Elaboración propia

Pilares del nuevo sistema:

1.La igualdad natural de todos los hombres.

2.Igualdad capacidad jurídica.

3.La imparcial aplicación del derecho a todos los hombres.

\section{Formulación teórica del principio:}

Según Smith, "Los ciudadanos de cualquier Estado deben contribuir al sostenimiento del gobierno, en cuanto sea posible, en proporción a sus respectivas aptitudes; es decir, en proporción a los ingresos que disfrutan bajo la protección estatal"

Orden institucional de Francia anterior a la revolución (Sánchez Viamonte).

\section{Ilustración No 2}

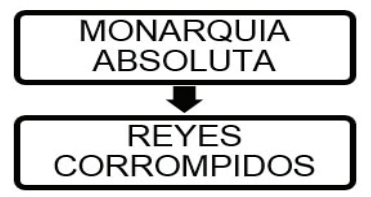

Fuente: Elaboración propia

En ese contexto institucional, el régimen fiscal se caracterizó por la más absoluta desigualdad. Ningún 
tributo era general para todos los súbditos, ni común a todo el reino (Saboul, 1983).

Característica: La más absoluta desigualdad, ningún impuesto era general para todos los súbditos, ni común a todo su reino.

LA NOBLEZA: Gozaba de privilegios de todo orden aproximadamente eran 350,000 personas. La nobleza constituía un monopolio de acceso a los más altos cargos públicos.

EL CLERO: Poseedor de extensos dominios, se encontraba al margen de la tributación 120,000 personas. Contribuía al sostenimiento de gastos del Estado mediante aportes voluntarios, considerados mínimos en relación con las rentas que percibían.

EL TERCER ESTAMENTO: Se denominaba desde el siglo XV el Tercer Estado, y estaba integrado por la mayoría de la población 24 millones de personas, de las cuales el $80 \%$ eran campesinos y el $20 \%$ burgueses.

El Tercer Estado, en especial los campesinos, absorbía el mayor peso de los tributos (Spisso).

Ilustración No 3

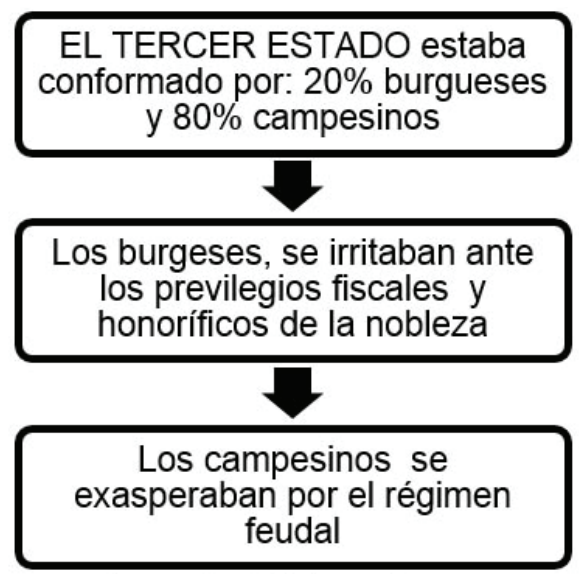

Fuente: Elaboración propia

Tal estado de cosas originó una reacción contraria a los privilegios e inmunidades fiscales, que quedó consagrada en la DECLARACIÓN DE LOS DERECHOS DEL HOMBRE Y DEL CIUDADANO.

\section{GENERALIDAD: IGUALDAD ANTE LA LEY}

El principio de igualdad ante la ley surge como una reacción al sistema de privilegios y discriminaciones. Más que propiciar una verdadera igualdad entre todas las personas, el principio persigue acabar con situaciones de desigualdad. Todas las personas son consideradas iguales ante la ley y titulares de los mismos derechos.

El vocablo "igualdad" se identifica con el de "generalidad”, y responde a la más clásica formulación del principio, en el sentido de igualdad ante la ley.

Neumark (1974) afirmaba que "el principio de generalidad de la imposición exige que, por una parte, todas las personas (naturales y jurídicas) - en tanto tengan capacidad de pago y queden tipificadas por una de las razones legales que dan nacimiento a la obligación tributaria, sin que se tengan en cuenta para ello, criterios extraeconómicos, tales como: nacionalidad, estamento y clases sociales, religión, raza, etc. sean sometidas al gravamen tributario y que, por otra parte, no se admitan en el marco de un impuesto en particular otras excepciones a la obligación tributaria subjetiva y objetiva que las que parezcan inexcusables por razones de política económica, social, cultural, sanitarias o por imperativos de la técnica tributaria"

\section{LA IGUALDAD COMO BASE DEL TRIBUTO}

La igualdad como base de los tributos, como medida de la obligación, significa que cada uno deberá contribuir en forma proporcional y progresiva a su capacidad económica.

Decir que todos deben contribuir no implica que no habrá excepciones, ya que la causa de la obligación de contribuir es la capacidad económica; y sin ella, no existe constitucionalmente obligación de tributar.

Vemos entonces, cómo generalidad y capacidad económica pueden ser vistas como especificaciones del principio de igualdad.

\section{DISTRIBUCIÓN DE LAS CARGAS PÚBLICAS. PROPORCIONALIDAD Y PROGRESIVIDAD}

El deber de los individuos de contribuir al sostenimiento de los gastos públicos debe corresponder a la capacidad económica de aquellos. Se presenta así, la capacidad contributiva como presupuesto legitimador del tributo. No puede haber imposición ante la inexistencia de capacidad económica, y no puede dejar de existir un tributo donde haya capacidad contributiva. 
La capacidad contributiva desempeña, un rol estelar, básico, aunque no exclusivo, que aplica con espíritu de justicia el principio de igualdad.

Si existe capacidad contributiva entonces se aplica el principio tributario de igualdad, presentándose así la capacidad contributiva como presupuesto legitimador del tributo.

La distribución de las cargas públicas debe ser estructurada en forma proporcional y progresiva a la capacidad económica de los sujetos pasivos del tributo.

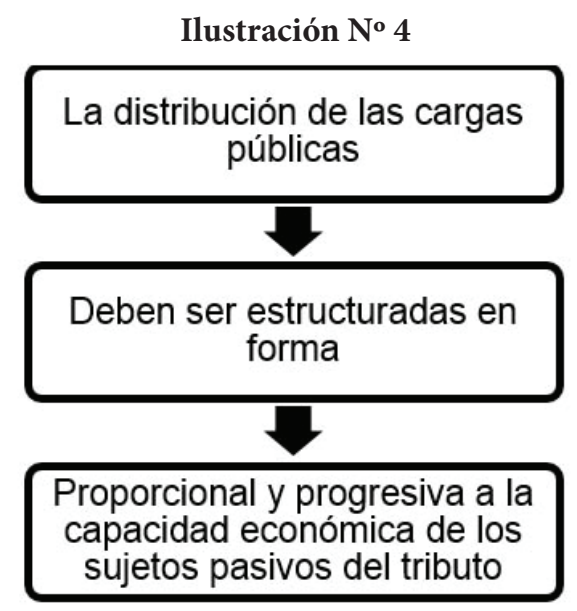

Fuente: Elaboración propia

La igualdad, por un lado, la proporcionalidad y la progresividad, por el otro, no son principios distintos, sino que constituyen una exigencia ineludible de aquella. La proporcionalidad y la progresividad más que principios propiamente dichos, son técnicas mediante las cuales se satisfacen el principio de igualdad como inspirador del sistema tributario, al servicio de una efectiva igualdad de hecho. (Spisso, Derecho Constitucional tributario).

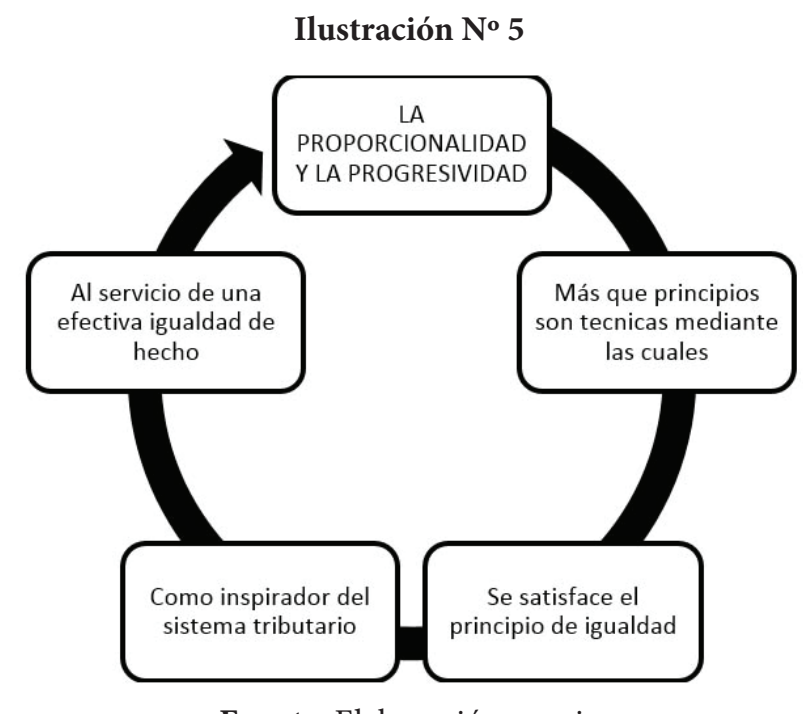

Fuente: Elaboración propia

La igualdad tributaria no puede ser entendida en sentido de igualdad matemática, es decir, que todos paguen la misma contribución.

La igualdad impone, cuanto menos, que sea proporcional a la capacidad económica.

La igualdad entre dos contribuyentes que se encuentran en situación de igualdad relativa, sólo se mantiene si la situación de uno respecto del otro, no se altera tras el pago de sus impuestos, lo cual exige una tributación progresiva, de forma tal que ambos experimenten un sacrificio proporcional en relación con la utilidad derivada de sus respectivos niveles de renta. (Lejeune Valcaccel, Aproximación al principio constitucional de igualdad tributaria).

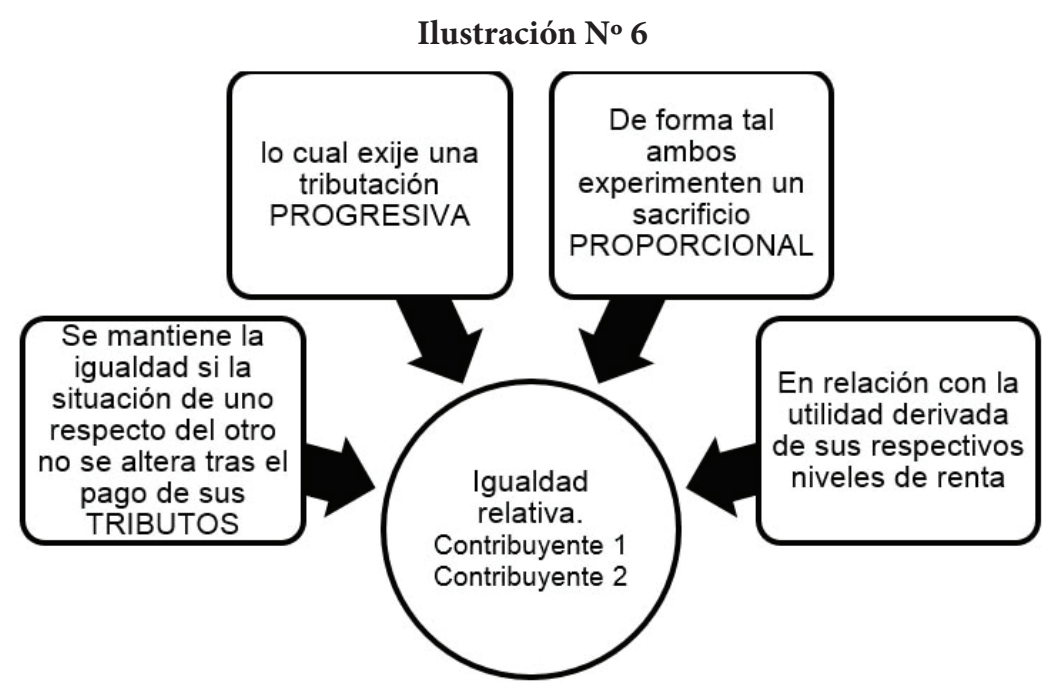

Fuente: Elaboración propia 


\section{SOCIEDAD DE PERSONAS}

Relación contractual basada en un convenio, escrito, oral o implícito, entre dos o más personas que combinan sus recursos y actividades en una empresa mancomunada y participan en grado variable y por convenio específico en la administración y en las utilidades o pérdidas. (Cooper, 2013)

\section{SOCIEDAD}

Conjunto de personas que se relacionan entre sí, de acuerdo a unas determinadas reglas de organización jurídicas y consuetudinarias, y que comparten una misma cultura o civilización en un espacio o un tiempo determinado. (Larousse, 2012)

\section{LEY DEL EQUILIBRIO FINANCIERO DEL PRESUPUESTO DEL SECTOR PÚBLICO 2014}

En la Ley del equilibrio financiero del presupuesto del sector público para el año fiscal 2014.

El Artículo $\mathrm{N}^{\circ} 1$ señala lo siguiente:

Recursos año fiscal 2014 S/. 118934253913 (Recaudación Total).

Fuente de Financiamiento S/. 82977000000 Recursos Ordinarios 70\%.

Los recursos ordinarios se refiere a los tributos recaudados por el Estado. (Legales, 2013).

\section{SUNAT (Superintendencia de Aduanas y de Administración tributaria)}

Según la Superintendencia Nacional de Aduanas y de Administración Tributaria (SUNAT) la recaudación acumulada real del Impuesto General a las Venta IGV (impuesto indirecto) aumentó en 5.2\% de enero a junio 2014 y el Impuesto a la Renta (impuesto directo) alcanzó 3.2\% (SUNAT, 1997 - 2012).

\section{CONCLUSIONES}

1. Los impuestos indirectos que origina los ingresos corrientes del Estado peruano, impacta en mayor grado a los estractos sociales de menores ingresos, generando mayores desigualdades sociales.

2. La SUNAT no considera el concepto de Responsabilidad Social, como ente recaudador tributario, lo que origina mayores brechas sociales.

\section{RECOMENDACIONES}

1. Invertir en ciudades más pobres del Perú con la finalidad de reducir diferencias; así también, dismunuir los impuestos indirectos (Impuesto General a las Ventas ) a largo plazo.

2. La SUNAT debe considerar la Responsabilidad Social como una forma ética de gestión que implica la inclusión de las expectativas de todos los grupos de interés.

\section{REFERENCIAS BIBLIOGRÁFICAS}

1. COOPER, W. (2013). Diccionario Kohler para contadores. México: Limusa.

2. LAROUSSE. (2012). El pequeño larousse ilustrado 2013. Barcelona: Larousse.

3. LEGALES, N. (2 de Diciembre de 2013). Ley $\mathrm{N}^{\circ}$ 30115. El Peruano, pág. 508184.

4. LEJEUNE, E. (s.f.). Aproximación al principio constitucional de igualdad tributaria. En seis estudios sobre derecho constitucional e internacional tributario. EDERSA.

5. MIDIS, M.d. (s.f.). MIDIS. Recuperado el 20 de Octubre de 2014, de Ministerio de Desarrollo e Inclusión Social : http://www.midis.gob.pe/ index.php/es/

6. NEUMARK, F. (1974). Principios de la imposición. Madrid: Instituto de estudIos fiscales.

7. SABOUL A. (1983). La Revolución Francesa, p. 71. Madrid.

8. SANCHEZ, C. (s.f.). Las instituciones políticas en la historia universal, p. 53.

9. SMITH, A. (s.f.). Investigación sobre la naturaleza y causas de la riqueza de las naciones, libro $\mathrm{V}$, cap. II, parte II.

10. SPISSO, R. R. (s.f.). Crisis terminal del régimen fiscal en el proceso de desinstitucionalización de la República, Derecho Tributario, t. III, p. 7.

11. SPISSO, R. R. (s.f.). Derecho Constitucional tributario. Buenos Aires: Lexinexis Argentina S.A.

12. SUNAT. (1997 - 2012). Estadística y Estudio. Recuperado el 20 de Octubre de 2014, de http:// www.sunat.gob.pe/estadisticasestudios/ 